\title{
Interference Analysis Status and Plans for Aeronautical Mobile Airport Communications System (AeroMACS)
}

Interference issues related to the operation of an aeronautical mobile airport communications system (AeroMACS) in the C-Band (specifically $5091-5150 \mathrm{MHz}$ ) is being investigated. The issue of primary interest is co-channel interference from AeroMACS into mobile-satellite system (MSS) feeder uplinks. The effort is focusing on establishing practical limits on AeroMACS transmissions from airports so that the threshold of interference into MSS is not exceeded. The analyses are being performed with the software package Visualyse Professional, developed by Transfinite Systems

Limited. Results with omni-directional antennas and plans to extend the models to represent AeroMACS more accurately will be presented. These models should enable realistic analyses of emerging AeroMACS designs to be developed from NASA Test Bed, RTCA 223, and European results. 


\title{
Interference Analysis Status and Plans for Aeronautical Mobile Airport Communications System (AeroMACS)
}

\author{
Robert J. Kerczewski and Jeffrey D. Wilson, \\ NASA Glenn Research Center
}

ICNS 2010 Conference 11-13 May 2010 


\section{Introduction}

\section{AeroMACS}

- Aeronautical Mobile Airport Communications System (AeroMACS) is being developed to provide next-generation broadband, networked communications for airport surface applications - safety critical, non-critical and advisory (ATC, AOC, AAC, airport operations)

- AeroMACS is based on IEEE 802.16e/802.16-2009 standards

- RTCA Special Committee 223 is working to develop standards for AeroMACS, an "aviation profile" derived from the IEEE 802.16

- Key technical parameters are under investigation, such as security issues, channelization of the 5091-5150 MHz allocation, interference issues, transmit power limitations, etc. 


\section{Introduction}

AeroMACS Interference Analysis

- The work described in the presentation addresses interference issues for the AeroMACS standard under development within RTCA SC-223

- AeroMACS transmit power limitations need to be determined in order to prevent interference from AeroMACS into other existing systems

- An analytical capability is needed to enable NASA to contribute technically to this effort:

- Enable required analyses to be performed in coordination with other organizations, within an RTCA sub group

- Complementary to other capabilities that will be employed, such as the Mitre-CAASD models

- Provide validation of results, increase confidence level for key results

- This presentation describes NASA Glenn Research Center's progress in developing and validation this capability 


\section{AeroMACS Interference Issues}

-Relevant 5091- $5150 \mathrm{MHz}$ in-band systems that need to be considered:

- Aeronautical Radionavigation Service (ARNS)

- Aeronautical Mobile Service (AMS) (Aeronautical Security (AS))

- Aeronautical Mobile Telemetry (AMT)

- Aeronautical Mobile (Route) Service (AM(R)S)

- Fixed Satellite Service (FSS) (Mobile Satellite Service (MSS) feeder uplink)

- Issue of immediate interest is interference from AeroMACS into the MSS feeder uplink

- This effort will focus on establishing practical limits on AeroMACS transmissions from airports so that the threshold of interference into MSS is not exceeded 


\section{Interference Analysis Modeling (1)}

Interference modeling is being performed with Visualyse Professional

Version 7 software from Transfinite Systems Limited (UK)

Modeling Procedure:

1. Define antennas

- Single beam, multiple beam, spot beam patterns

- Beam shape can be circular, elliptical, or shaped with userdefined gain dependence on azimuth and elevation angles

2. Locate stations

- Locations of transmitters and receivers

-Types: Earth, Mobile, Maritime, Aeronautical, GSO Satellite, non-GSO Satellite

3. Specify carriers

- Frequency, bandwidth, polarization

- Spectrum masks to calculate adjacent channel and out of band interference levels 


\section{Interference Analysis Modeling (2)}

Modeling Procedure (continued):

4. Set up propagation environment

- Choose loss model from ITU-R menu

- Can include rain attenuation, atmospheric losses

5. Set up links

- Connect stations

- Form communications paths by combining stations carriers, propagation, interference criteria

- Types: fixed, receive, transmit, dynamic

6. Set up interference paths

- Select victim link(s)

- Select interfering link(s)

7. Specify output desired, run, and analyze results

- Detailed statistics

- Visual, graphical output

- Monte Carlo runs with varying parameters 


\section{Benchmark Case}

Reference: Hoh, Gheorghisor, and Box, 2005, Feasibility Analysis of 5091-5150 MHz Band Sharing by ANLE and MSS Feeder Links, MITRE-CAASD Report MP $05 W 0000083$

Key Aspects:

- Investigated co-channel interference from ANLE (Airport Network and Location Equipment / now referred to as AeroMACS) system to non-geostationary mobile-satelliteservice (MSS) feeder uplinks

-Assumed one transmitter with omni-directional antenna at each of 497 major airports in contiguous United States

-Worst case scenario: all transmitters on $100 \%$ of time

- Calculated aggregate interference power at LEO from each airport antenna:

$$
P_{r}(d B W)=P_{t}+G_{t}-L_{c}+G_{r}-L_{\text {free }}(d)-L_{\text {feed }}-L_{p}+B_{f}-30
$$




\section{Benchmark Parameters (from Hoh, et al.)}

- $P_{\mathrm{t}}=$ transmitter power

$$
=38.6 \mathrm{dBm}(8.6 \mathrm{~dB}=7.2 \mathrm{~W})
$$

- $\mathrm{G}_{\mathrm{t}}=$ transmit antenna gain

$$
=8 \mathrm{dBi} \text { peak (see figure) }
$$

- $\mathrm{L}_{\mathrm{c}}=$ cable/line loss $=1 \mathrm{~dB}$

- $\mathrm{G}_{\mathrm{r}}=$ receive antenna gain

$$
=6 \mathrm{dBi} \text { peak (see figure) }
$$

- $\mathrm{L}_{\text {free }}=$ free-space path loss $(\mathrm{dB})$

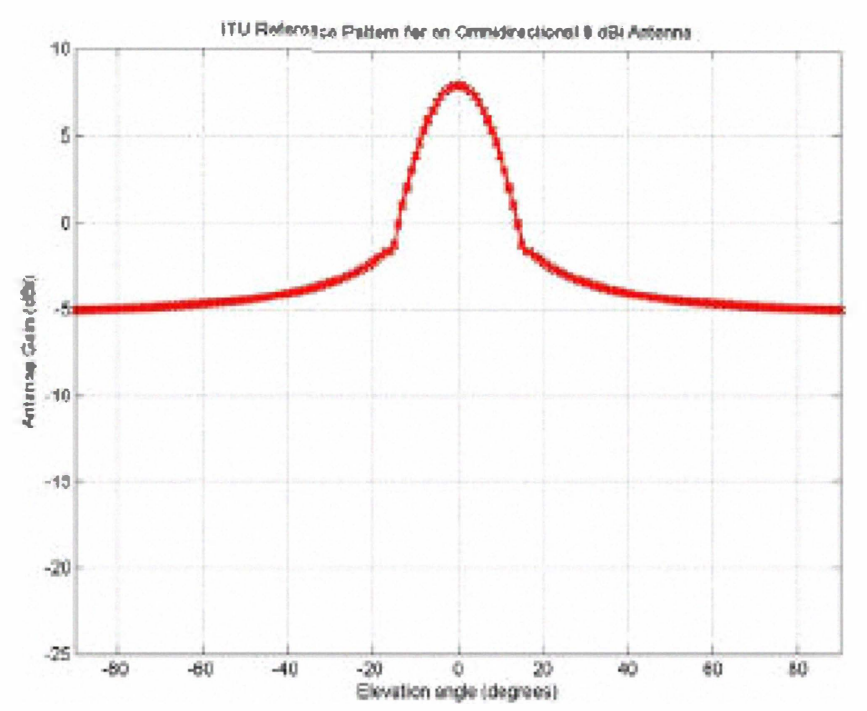

- $\mathrm{L}_{\text {feed }}=$ feed loss $=2.9 \mathrm{~dB}$

- $L_{p}=$ polarization discrimination $=1 \mathrm{~dB}$

- $B_{f}=$ bandwidth factor

$$
\begin{aligned}
& =10^{*} \log \left(\mathrm{B}_{\mathrm{LEO}} / \mathrm{B}_{\mathrm{ANLE}}\right) \\
& =10^{*} \log (1.23 / 20)=-12.1 \mathrm{~dB}
\end{aligned}
$$

- $d=$ distance between transmitter and LEO satellite receiver $=(1414+\mathrm{km})$

Transmit antenna gain pattern

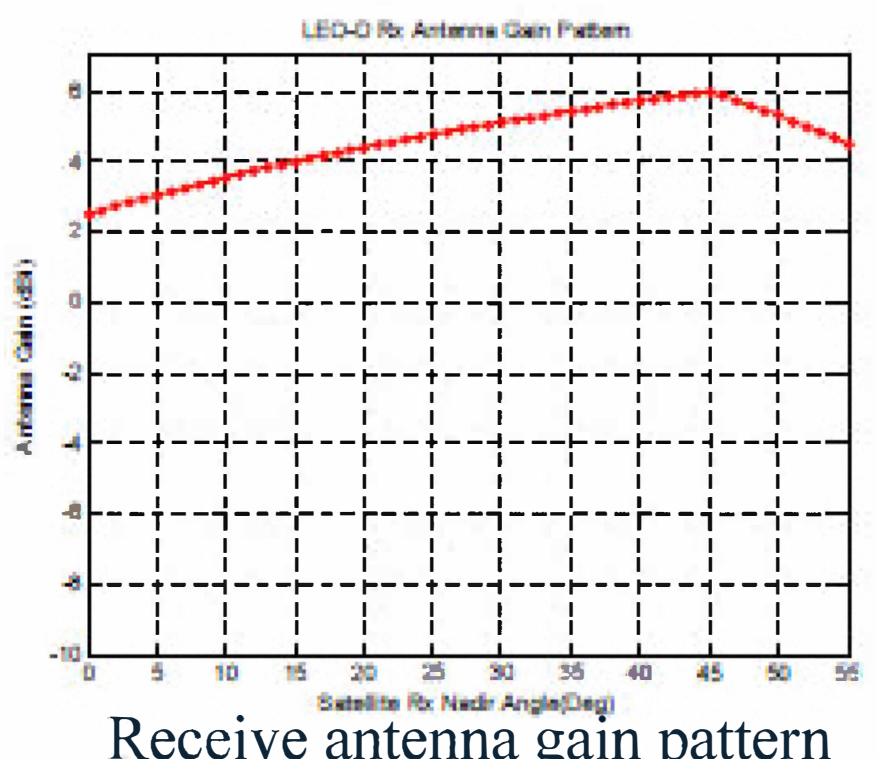




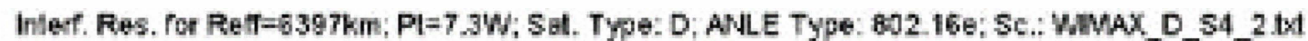

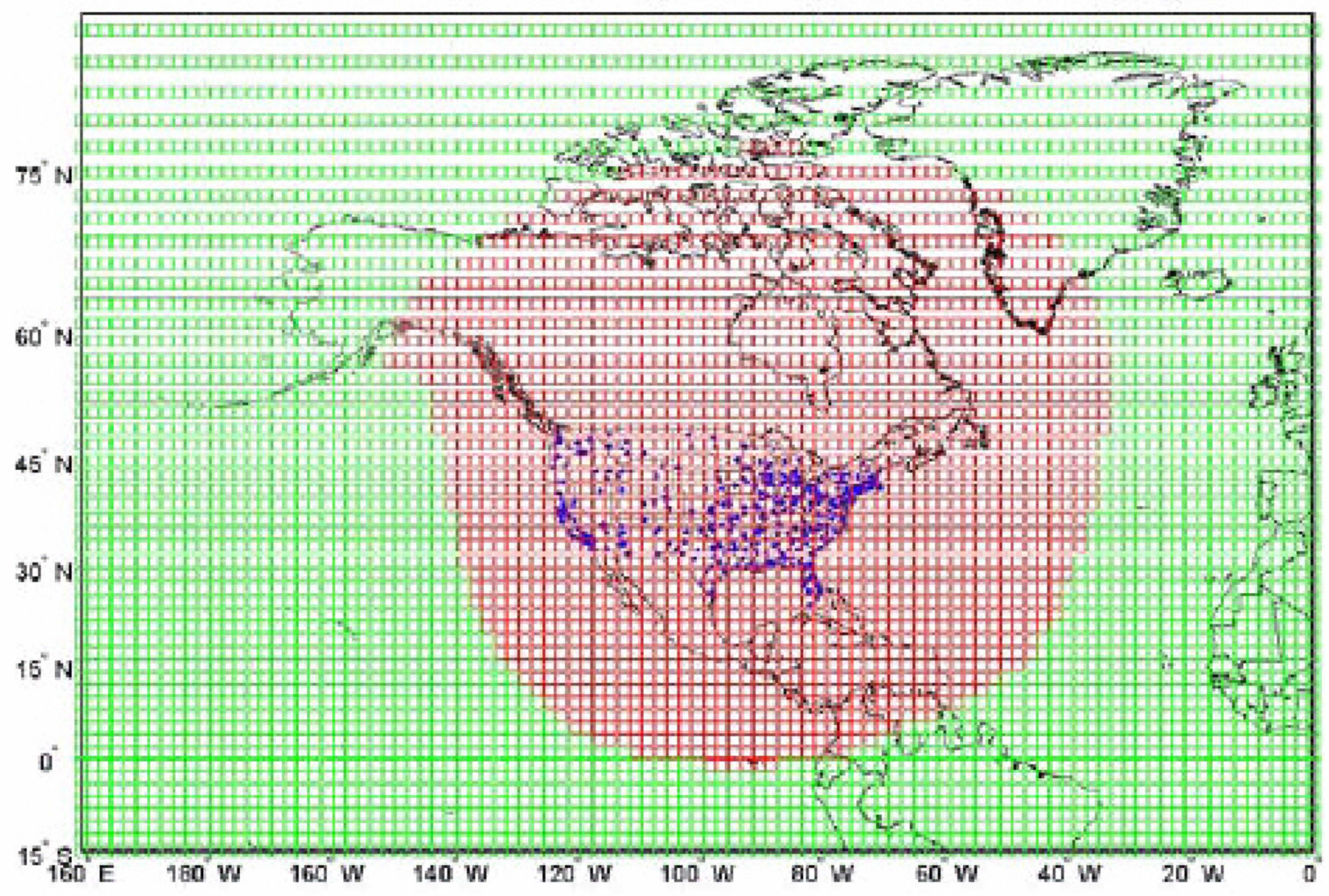

- Aggregate interference power at LEO (1414 km) from 497 airports

- Threshold ( - $155.5 \mathrm{dBW}$ ) exceeded in red area*

- Maximum interference power of $-150.0 \mathrm{dBW}$ at $67^{\circ} \mathrm{N} 104^{\circ} \mathrm{W}$

*Note threshold definition has since been tightened to $-157.3 \mathrm{dBW}$ corresponding to $2 \%$ increase of satellite receiver's noise temperature 


\section{Visualyse Results Comparison}

Hoh, et al.:

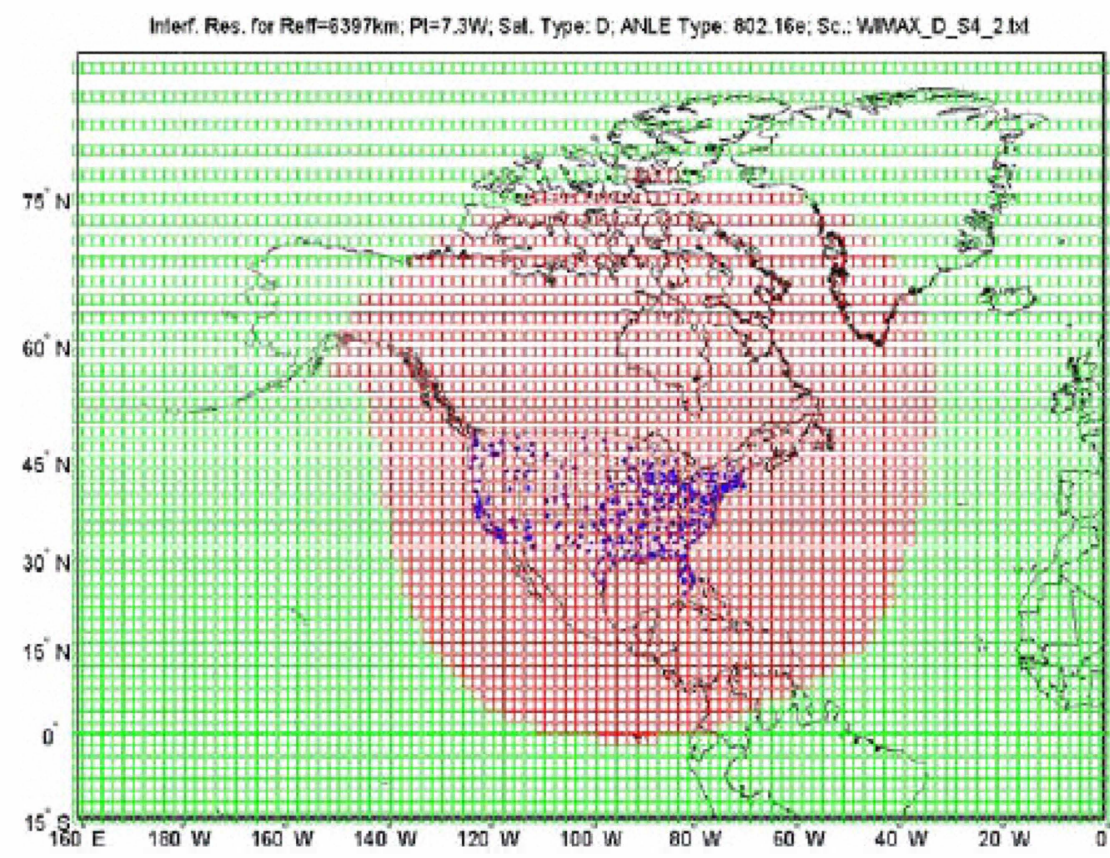

- Red/green border $=$ threshold

- Max interference $=-150.0 \mathrm{dBW}$

- Max location $=67^{\circ} \mathrm{N} 104^{\circ} \mathrm{W}$
Visualyse:

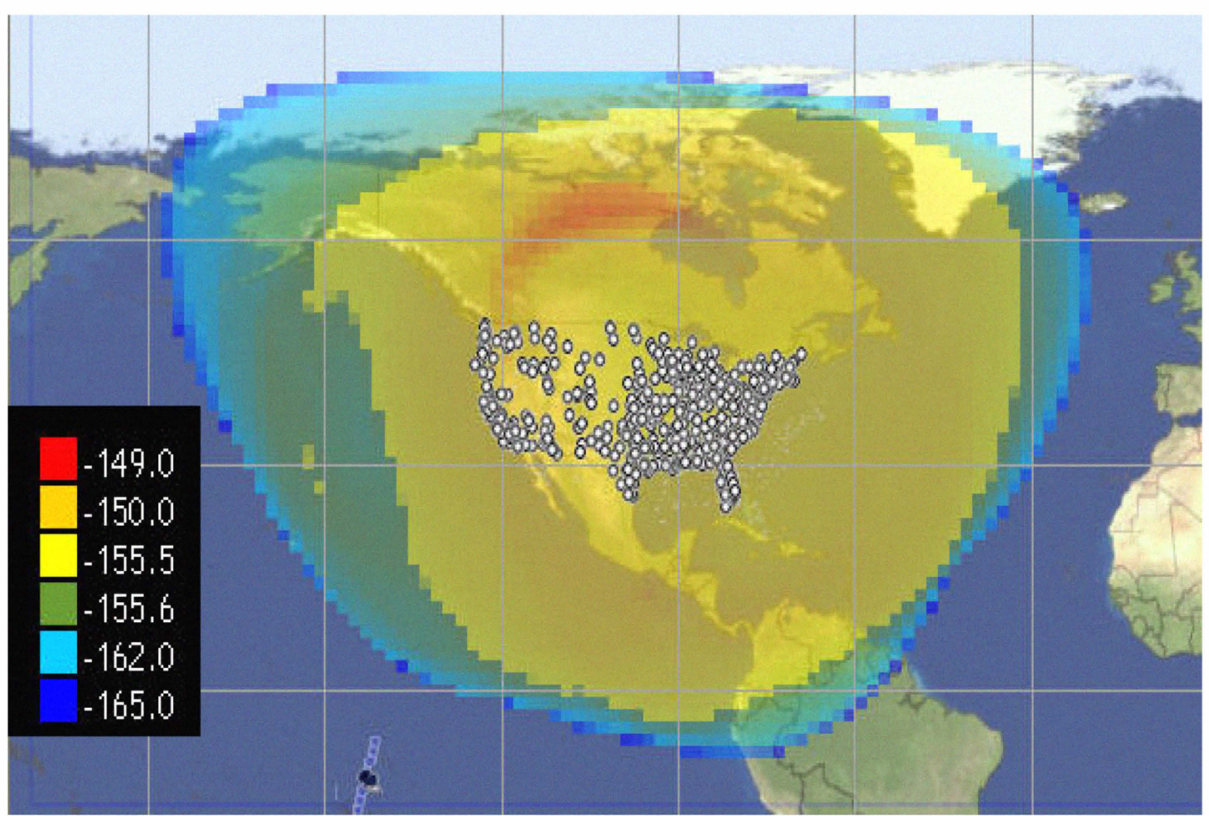

- Yellow/green border $=$ threshold

- Max interference $=-149.3 \mathrm{dBW}$

- Max location $=64^{\circ} \mathrm{N} 105^{\circ} \mathrm{W}$ 


\section{Visualyse Global View}

- Corresponding global view shows widespread area of threshold violation (yellow and red) in this worst case scenario

- Confirms need to investigate sectoral antennas as in MITRECAASD study

(Gheorghisor, Hoh, and Leu, et al., 2009, Analysis of ANLE Compatibility with MSS Feeder Links, MITRE-CAASD Report MTR090458)

- Max interference power over northern Canada and secondary max over Central America due

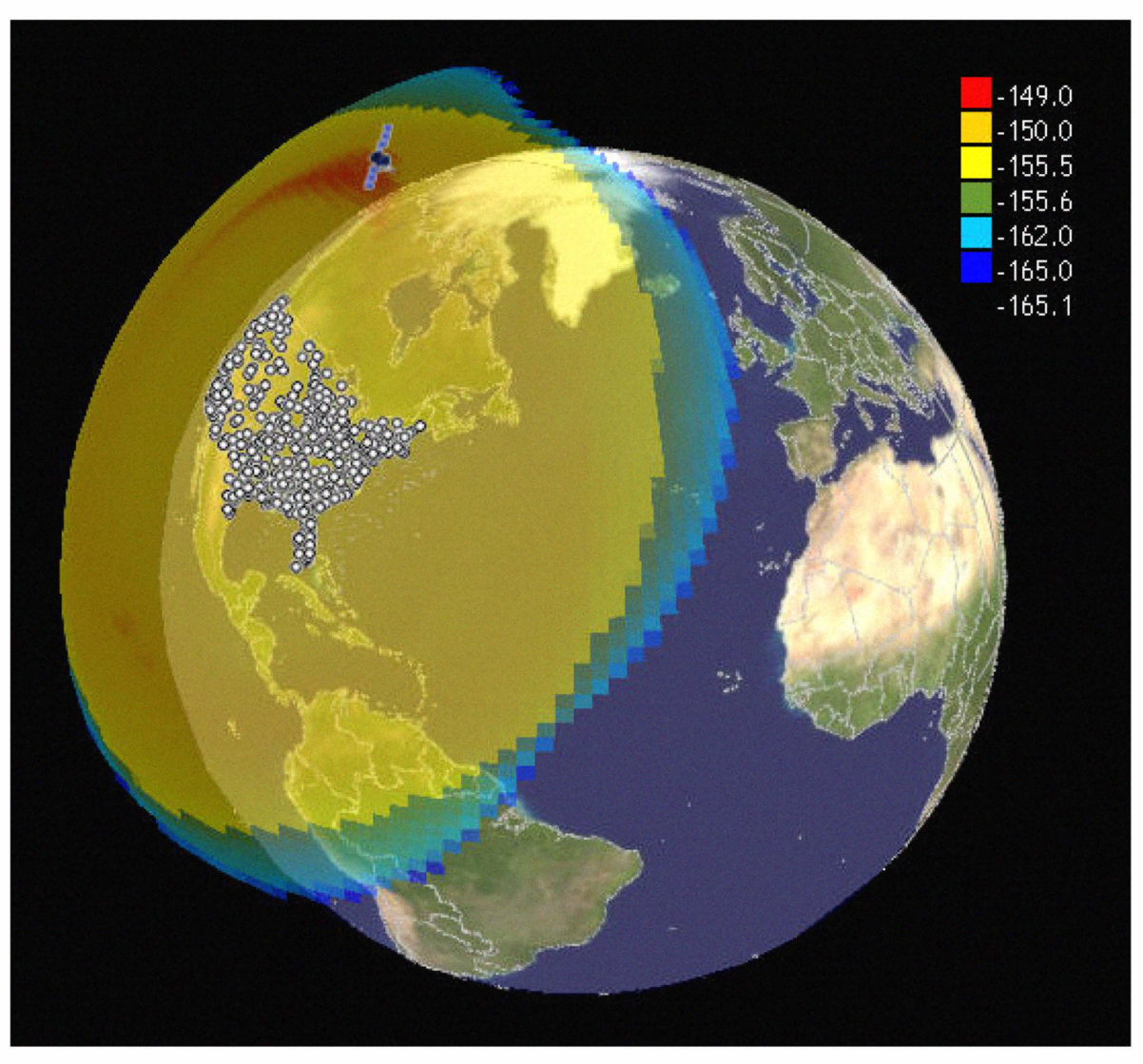
to maximum transmitting gain of antennas in horizontal direction 


\section{Interference Power for Single Airport Model}

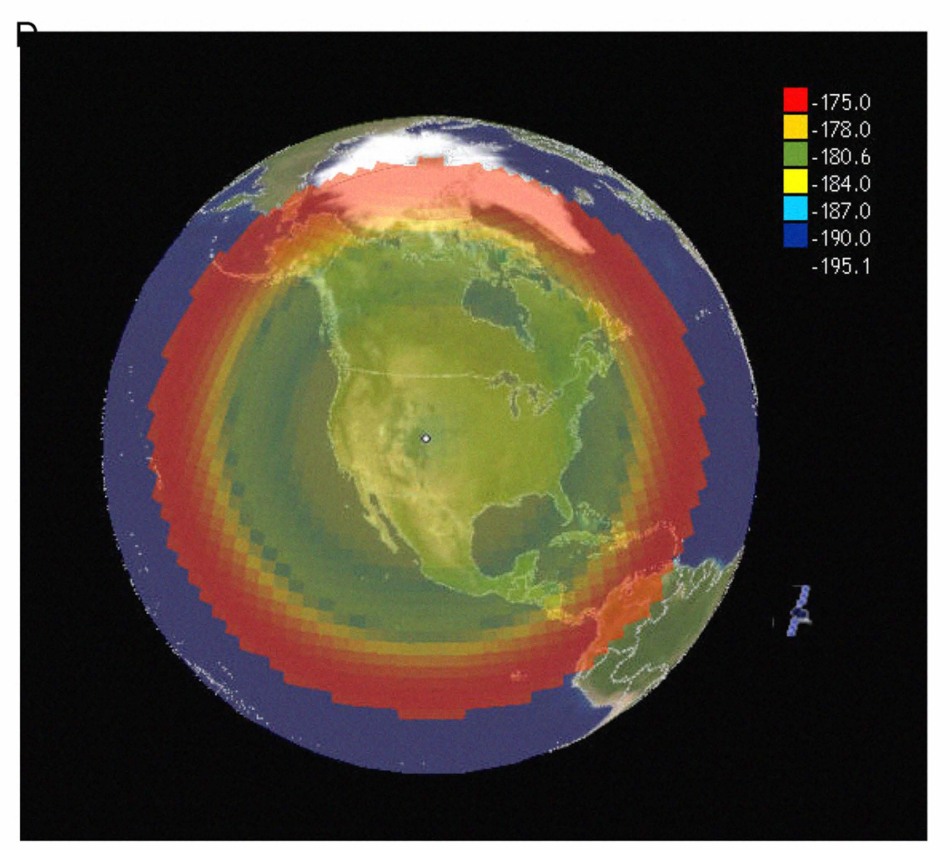

Denver (sea level)

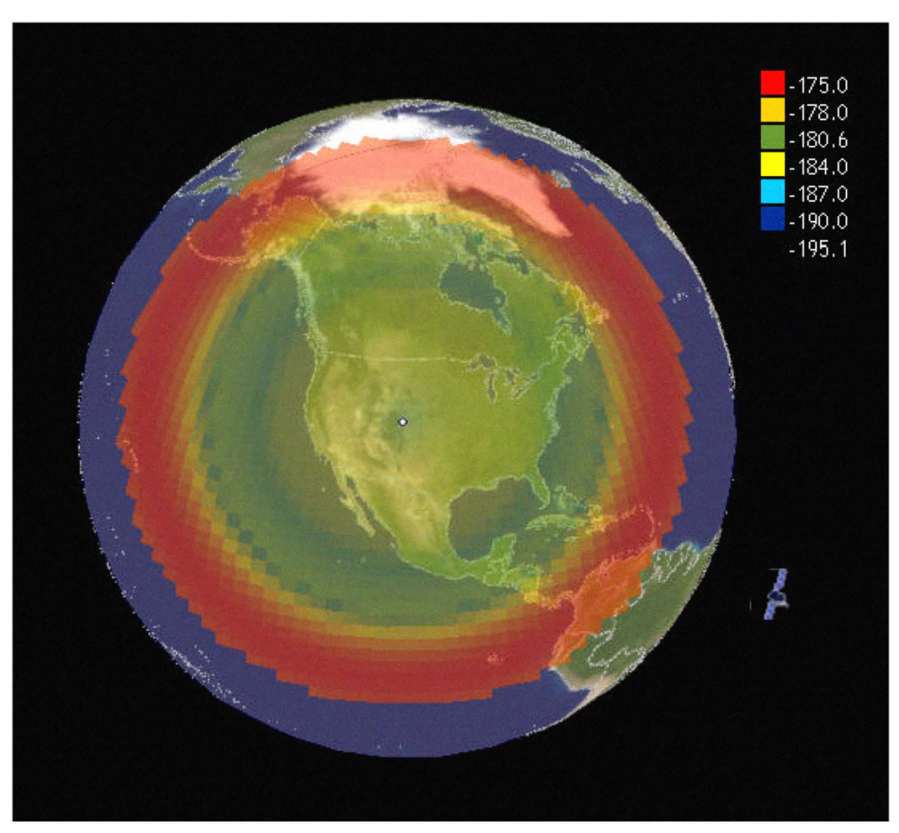

Denver ( 5431 feet)

- Max interference power -175.5 dBW in both

- Station elevation $(1.6 \mathrm{~km})$ not very important at LEO $(1414 \mathrm{~km})$ 


\section{Interference Power for 703 Airports Model}

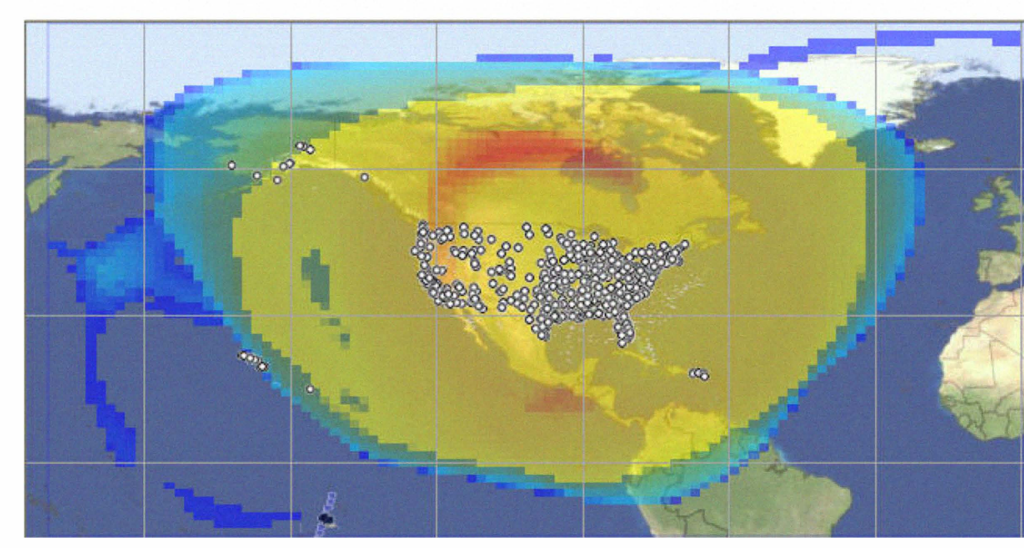

703 Airports

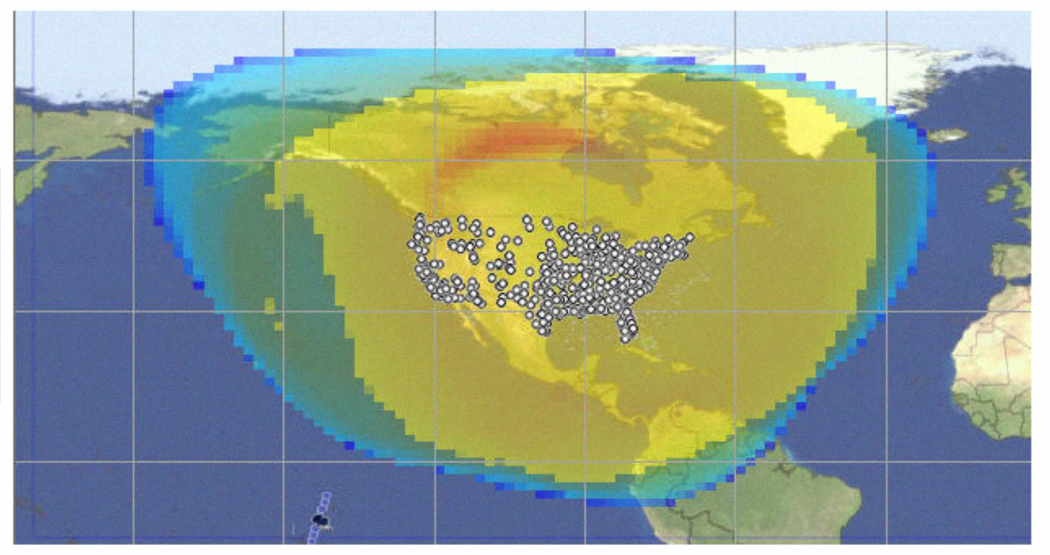

497 Airports

- Model includes 703 FAA towered airports and heliports, including Alaska, Hawaii, and Caribbean, from FAA data base

- More that the 497 airports considered in Hoh, et al.

- Interference threshold violation extends further into Pacific.

- Maximum interference power increases only by $0.3 \mathrm{dBW}$. 
National Aeronautics and Space Administration

\section{Wasilla Globalstar Uplink Movie}

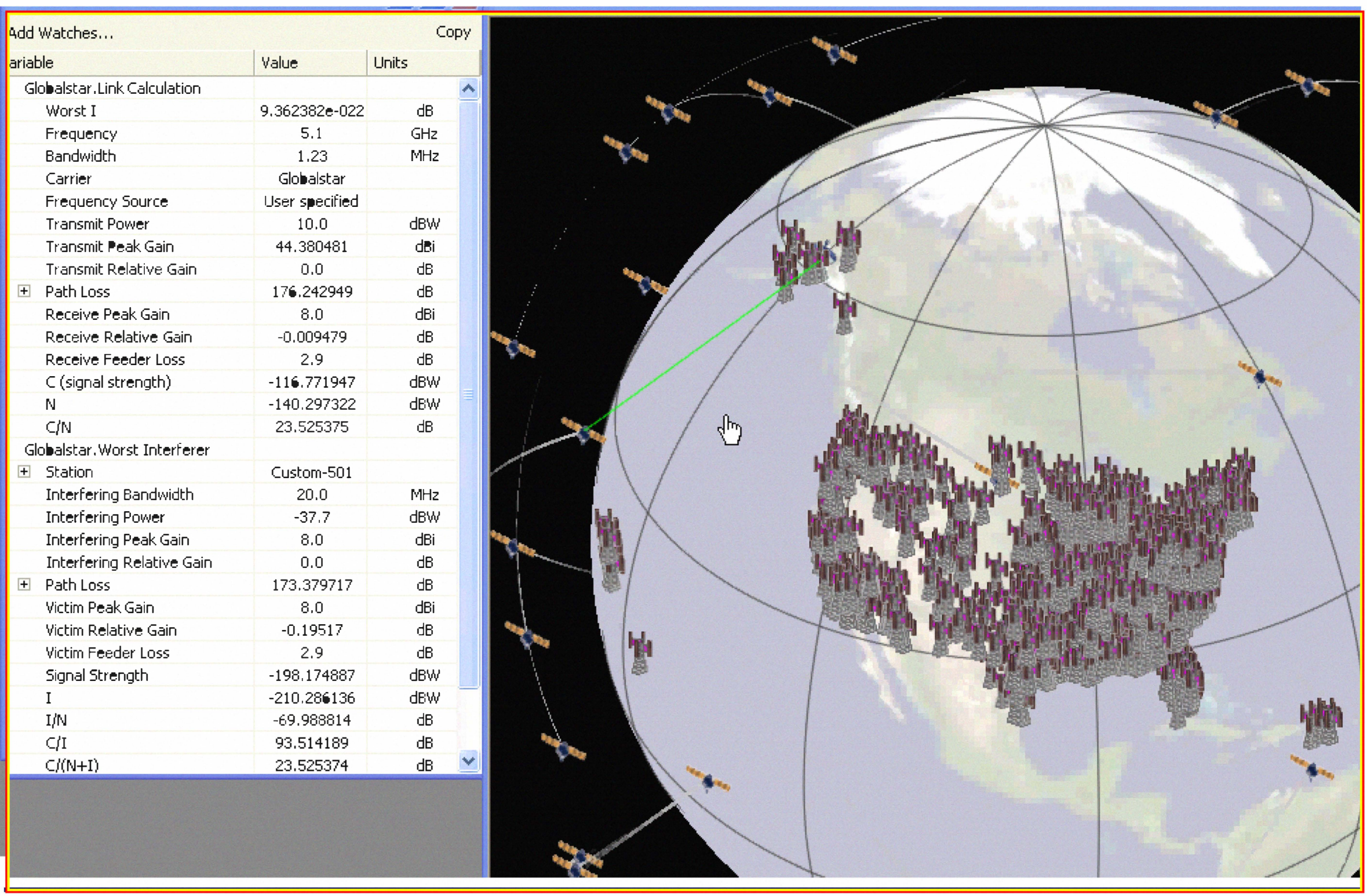




\section{Future Plans}

- Year 2010 plans to increase complexity and realism of interference models:

- Multi-sector antennas.

- Airport surface network with multiple base and subscriber stations.

- Year 2011 and beyond potential plans:

- Interference from AeroMACS into adjacent frequency bands.

- Co-channel and adjacent band interference into AeroMACS.

- Geographically close AeroMACS implementations.

- Multiple stations, frequency re-use, and multipath signal propagation effects in single airport. 


\section{Conclusions}

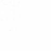

- Need to establish practical limits on AeroMACS transmissions from airports so that the threshold of interference into MSS and other C-band systems is not exceeded

- Enable the development of standards under RTCA SC-223

- Analyses have been started with Visualyse, a powerful and flexible interference analysis software tool.

- Visualyse results with omni-directional antenna transmitters agree very well with results from MITRE-CAASD.

- Near term plans to improve model realism with multi-sector antennas and subscriber stations. 